\title{
Alzheimer's Disease Classification With a Cascade Neural Network
}

\author{
Zeng You ${ }^{1,2+}$, Runhao Zeng ${ }^{2+}$, Xiaoyong Lan ${ }^{1+}$, Huixia Ren ${ }^{1,3}$, Zhiyang You ${ }^{1,2}$, Xue Shi ${ }^{1}$, \\ Shipeng Zhao ${ }^{1,2}$, Yi Guo $^{1 *}$, Xin Jiang ${ }^{4 *}$ and Xiping $\mathrm{Hu}^{2 *}$ \\ 'Department of Neurology, Shenzhen People's Hospital, The First Affiliated Hospital of Southern University of Science and \\ Technology, The Second Clinical Medical College of Jinan University, Shenzhen, China, ${ }^{2}$ Shenzhen Institutes of Advanced \\ Technology, Chinese Academy of Sciences, Shenzhen, China, ${ }^{3}$ The First Affiliated Hospital, Jinan University, Guangzhou, \\ China, ${ }^{4}$ Department of Geriatrics, Shenzhen People's Hospital, The First Affiliated Hospital of Southern University of Science \\ and Technology, The Second Clinical Medical College of Jinan University, Shenzhen, China
}

\section{OPEN ACCESS}

Edited by:

Ying Li,

Zhejiang University, China

Reviewed by:

Laisen Nie,

Northwestern Polytechnical University,

China

Weijing Qi,

Chongqing University of Posts and

Telecommunications, China

${ }^{*}$ Correspondence:

Yi Guo

xuanyi_guo@163.com

Xin Jiang

jiangxinsz@163.com

Xiping $\mathrm{Hu}$

xp.hu@siat.ac.cn

tThese authors have contributed equally to this work

Specialty section: This article was submitted to

Digital Public Health,

a section of the journal

Frontiers in Public Health

Received: 17 July 2020 Accepted: 28 September 2020

Published: 03 November 2020

Citation:

You Z, Zeng R, Lan X, Ren H, You Z,

Shi $X$, Zhao S, Guo Y, Jiang $X$ and

Hu X (2020) Alzheimer's Disease

Classification With a Cascade Neural

Network

Front. Public Health 8:584387.

doi: 10.3389/fpubh.2020.584387
Classification of Alzheimer's Disease (AD) has been becoming a hot issue along with the rapidly increasing number of patients. This task remains tremendously challenging due to the limited data and the difficulties in detecting mild cognitive impairment (MCl). Existing methods use gait [or EEG (electroencephalogram)] data only to tackle this task. Although the gait data acquisition procedure is cheap and simple, the methods relying on gait data often fail to detect the slight difference between $\mathrm{MCl}$ and $\mathrm{AD}$. The methods that use EEG data can detect the difference more precisely, but collecting EEG data from both $\mathrm{HC}$ (health controls) and patients is very time-consuming. More critically, these methods often convert EEG records into the frequency domain and thus inevitably lose the spatial and temporal information, which is essential to capture the connectivity and synchronization among different brain regions. This paper proposes a cascade neural network with two steps to achieve a faster and more accurate AD classification by exploiting gait and EEG data simultaneously. In the first step, we propose attention-based spatial temporal graph convolutional networks to extract the features from the skeleton sequences (i.e., gait) captured by Kinect (a commonly used sensor) to distinguish between $\mathrm{HC}$ and patients. In the second step, we propose spatial temporal convolutional networks to fully exploit the spatial and temporal information of EEG data and classify the patients into $\mathrm{MCl}$ or $A D$ eventually. We collect gait and EEG data from 35 cognitively health controls, $35 \mathrm{MCl}$, and $17 \mathrm{AD}$ patients to evaluate our proposed method. Experimental results show that our method significantly outperforms other AD diagnosis methods (91.07 vs. 68.18\%) in the three-way $A D$ classification task $(\mathrm{HC}, \mathrm{MCl}$, and $\mathrm{AD})$. Moreover, we empirically found that the lower body and right upper limb are more important for the early diagnosis of AD than other body parts. We believe this interesting finding can be helpful for clinical researches.

Keywords: Alzheimer's disease, deep learning, automatic diagnosis, gait, EEG

\section{INTRODUCTION}

Alzheimer's disease (AD) is the most common cause of cognitive impairment and is one of the diseases with the highest incidence among the elderly. In 2006, 26.6 million people on the earth suffered from $\mathrm{AD}$, and the number is still rapidly increasing every year (1). More critically, $\mathrm{AD}$ has become the seventh leading cause of death (2). Conventional AD diagnosis methods often use scale 
screening and brain imaging equipment such as functional Magnetic Resonance Imaging (fMRI), Computer Tomography (CT), and Positron Emission Tomography (PET). These methods require experienced clinicians as well as exhaustive examinations.

Recently, many studies (3-9) have been conducted to reduce the diagnosis cost and shorten the diagnosis time by designing an $\mathrm{AD}$ classification system that is able to detect and classify $\mathrm{AD}$ automatically. However, it is challenging to classify AD precisely for the following reasons: on the one hand, the prodromal stage of $\mathrm{AD}$, namely mild cognitive impairment (MCI), has a light symptom, making it hard to detect; On the other hand, extracting robust features for $\mathrm{AD}$ detection is very challenging due to the limited volume of medical data.

Previous studies on AD classification exploit gait data (3, $10-17)$ due to the strong relationship between gait features and cognitive function (18-25). They often extract hand-crafted features from the input gait data (e.g., skeleton) and classify $\mathrm{AD}$ relying on these features. However, designing hand-crafted features for $\mathrm{AD}$ classification requires expert knowledge, and it is difficult to generalize the hand-crafted features to other tasks. Recently, some researchers $(12,13,15,16,26,27)$ attempt to conduct AD classification using EEG data. However, existing EEG-based methods often $(6,7)$ need to convert EEG data into frequency domain information and calculate the Power Spectral Density (PSD) features for classification. In this sense, these methods will inevitably lose the information in the spatial and time domains of EEG data, which, however, is very important for capturing the coherence and synchronizations among different brain regions. It is worth noting that existing methods use one modal only (gait or EEG data) and suffer from the following limitations: (1) as discussed in $(28,29)$, using gait data can accurately distinguish $\mathrm{HC}$ and patients but often fails to classify $\mathrm{MCI}$ and $\mathrm{AD}$, and (2) using EEG data can classify MCI and AD more accurately, but it is time-consuming to collect EEG data from both $\mathrm{HC}$ and patients.

We contend that considering the two modalities (i.e., gait and EEG data) simultaneously helps achieving faster and more accurate classification. To this end, we propose a cascade neural network with two steps for the early diagnosis of $\mathrm{AD}$ using both gait data and EEG data simultaneously. In the first step, we use gait data to classify $\mathrm{HC}$ and patients. For the purpose of reducing the psychological disturbance to the subject, we follow (10) to use the Kinect devices as the acquisition equipment to capture skeleton sequences. Regrading the non-Euclidean skeleton data, we propose to use attentionbased spatial temporal graph convolutional networks (ASTGCN) to model the relationships among body key points and automatically extract powerful features for distinguishing between $\mathrm{HC}$ and patients. In the second step, we use the original EEG data to distinguish MCI and AD patients further. Unlike other methods that convert EEG data to the frequency domain, we propose spatial temporal convolutional networks (ST-CNN) to directly extract the spatial and temporal features from original EEG data and use them to classify MCI and $\mathrm{AD}$. In this manner, the EEG data from $\mathrm{HC}$ are no longer required, saving a lot of data collection time. We collect a data set consisting of gait and EEG data from 35 cognitively health controls, $35 \mathrm{MCI}$ patients, and $17 \mathrm{AD}$ patients to evaluate our proposed method.

Our main contributions are summarized as follows:

- We propose a cascade neural network that uses both gait and EEG data to classify AD, which achieves a high accuracy rate with less manual participation. This is the first attempt to consider two modalities for $\mathrm{AD}$ classification to the best of our knowledge.

- We propose attention-based spatial temporal graph convolutional networks to automatically extract the features from gait data and leverage them to classify AD.

- Moreover, we also propose spatial temporal convolutional networks to fully extract the spatial and temporal features from the original EEG data in both space and time domains.

- The accuracy rate of our proposed cascade neural network in the three-way classification of $\mathrm{HC}, \mathrm{MCI}$, and $\mathrm{AD}$ reaches $91.07 \%$, which is much higher than the method using one modal $(68.18 \%)$. The accuracy of $\mathrm{HC}$ vs. MCI/AD is up to $93.09 \%$.

The rest of the paper is arranged as follows: Related work is concentrated on section 2; Section 3 details the proposed framework and the modules in it; Experimental results are exhibited in section 4; Section 5 concludes this paper.

\section{RELATED WORK}

Gait data has been used extensively to classify AD. Wang et al. (3) developed a device to collect the inertial signals of subjects. They designed an algorithm to leverage the inertial signals to detect and calculated the features of the stride. Then they selected the salient features to classify $\mathrm{HC}$ and $\mathrm{AD}$. The classification accuracy rates in the female and the male groups are 70.00 and $63.33 \%$, respectively. Choi et al. (29) compared the gait and cognitive function between the $\mathrm{HC}$ group and $\mathrm{MCI} / \mathrm{AD}$ groups. They found that gait features can distinguish MCI and $\mathrm{HC}$, while cognitive tests are suitable for distinguishing $\mathrm{AD}$ and $\mathrm{HC}$. The average detection rate of $\mathrm{AD}$ and $\mathrm{MCI}$ from $\mathrm{HC}$ using gait variables is $75 \%$. Seifallahi et al. (10) used Kinect to collect gait data, extracted, and screened the features. Then they used Support Vector Machines (SVM) to classify AD and HC. The classification accuracy rate is $92.31 \%$. Varatharajan et al. (4) used IoT devices to collect gait data and then extracted the features using the dynamic time warping (DTW) algorithm. The accuracy rate of classification is about $70 \%$. Although the above works achieve good performance, they all rely on handcrafted feature extraction, which cannot guarantee the full use of the implicit information in gait data, and the features designed for specific tasks cannot be applied to other general tasks. The attention-based spatial temporal graph convolutional networks we proposed can automatically extract gait data features and exploit the relationships among body joints.

EEG data is another important information that can be used to diagnose AD. Existing methods for the early diagnosis of $\mathrm{AD}$ using EEG data can be categorized into handcrafted feature based-methods and deep learning methods. Anderer 
et al. (12) and Pritchard et al. (13) input EEG markers into an ANN to perform a binary classification between $\mathrm{AD}$ and HC with an accuracy rate of $90 \%$. Trambaiolli et al. (15) extracted features based on coherence and used Support Vector Machines(SVM) to classify AD and HC, with 79.9\% accuracy. Rossini et al. (16) tested the IFAST procedure to classify HC and MCI, achieving $93.46 \%$ accuracy. These methods all require handcrafted feature extraction. In recent years, more and more deep learning methods have been applied to the classification of AD. Ieracitano et al. (6) calculated the PSD features of the subject's EEG data. They converted the PSD features into images, and then used the convolutional neural networks for the early diagnosis of $\mathrm{AD}$, achieving an accuracy of $89.8 \%$ in the binary classification and $83.3 \%$ in three-way classification. Bi and Wang (7) calculated the PSD features of EEG data, then used the feature representation method proposed by (30) to convert the PSD features into images. They designed a DCssCDBM with a multi-task learning framework, achieving an accuracy of up to 95.05\%. These deep learning methods all need to convert EEG data into frequency domain information. This way will lose the information in the spatial and temporal domains of EEG data, which is essential for capturing coherence and synchronization among different brain regions. We directly use the original EEG data containing both spatial and temporal information. We propose spatial temporal convolutional networks to extract the temporal and spatial implicit features of EEG data.

The methods mentioned above leveraged either gait data or EEG data only for the early diagnosis of $\mathrm{AD}$. The gait data collection procedure is simple, short in time, and easy to operate, but there is no significant difference in gait features between MCI and AD (29), and thus method relying on gait data cannot classify AD and MCI precisely. Conversely, EEG data can provide promising cues to classify $\mathrm{AD}$ and $\mathrm{MCI}$, but the acquisition process is complicated and takes a long time. We consider gait and EEG data simultaneously to achieve a fast and accurate classification of AD.

\section{PROPOSED METHOD}

Notation. Let $\mathcal{S}=\left\{s_{i}\right\}_{i=1}^{N_{s}}$ be the subject set that includes $N_{s}$ subjects, where $s_{i}$ represents the $i^{\text {th }}$ subject. Let $\mathcal{G}_{i}=\left\{g_{i}^{j}\right\}_{j=1}^{N_{g}}$ denote clip set where $N_{g}$ clips are sampled from the gait data of the $i^{\text {th }}$ subject $s_{i}$, where $g_{i}^{j}$ represents the $j^{\text {th }}$ clip. Let $\mathcal{E}_{i}=\left\{\varepsilon_{i}^{e}\right\}_{e=1}^{N_{e}}$ denote the epoch set containing $N_{e}$ epochs sampled from the EEG data of the $i^{\text {th }}$ subject $s_{i}$, where $\varepsilon_{i}^{e}$ represents the $e^{\text {th }}$ epoch.

Problem Definition. Given gait clip set $\mathcal{G}_{i}$ and EEG epoch set $\mathcal{E}_{i}$ of subject $s_{i}$, the classification of AD aims to map physiological signals, $\mathcal{G}_{i}$ and $\mathcal{E}_{i}$, into $\mathrm{HC}, \mathrm{MCI}$, and $\mathrm{AD}$ groups corresponding to the state of subject $s_{i}$. This task is very challenging due to the limited volume of data and the subtle differences among the three groups, especially for $\mathrm{HC}$ and MCI.

\subsection{Pipeline Overview}

Existing methods used either gait data or EEG data only for the classification of AD. However, as discussed in $(28,29)$, using gait data can accurately distinguish $\mathrm{HC}$ and patients, but the methods using gait data only often fail to classify MCI and AD. For the EEG data that are more sensitive to the differences between MCI and $\mathrm{AD}$, some studies used EEG data to classify AD. However, collecting EEG data from both $\mathrm{HC}$ and patients takes a lone time. We believe that combining the two is able to make the early diagnosis of $\mathrm{AD}$ faster and more accurately. This drives us to propose a cascade neural network for the early diagnosis of $\mathrm{AD}$ with both gait and EEG data.

Given gait $\operatorname{clip} g_{i}^{j}$ and EEG epoch $\varepsilon_{i}^{e}$ of subject $s_{i}$, we conduct the classification in two steps. Firstly, we use gait data to distinguish $\mathrm{HC}$ and $\mathrm{MCI} / \mathrm{AD}$ patients. In this step, we select key points from $g_{i}^{j}$ to form key-point skeleton sequences first. Then we input the key-point skeleton sequences into attention-based spatial temporal graph convolutional networks (AST-GCN) to extract features. Finally, we use these features to classify $\mathrm{HC}$ and $\mathrm{MCI} / \mathrm{AD}$ by a standard SoftMax classifier. We further distinguish AD from MCI with EEG epoch $\varepsilon_{i}^{e}$ in the second step. We input $\varepsilon_{i}^{e}$ into the spatial temporal convolutional networks (ST-CNN) to extract the implicit features in spatial and temporal domain. We then used the features extracted by ST-CNN for the binary classification of MCI vs. AD. In our method, the EEG data from $\mathrm{HC}$ are not required. The architecture of our proposed framework is shown in Figure 1.

\subsection{Attention-Based Spatial Temporal Graph Convolutional Networks}

Existing methods that use gait data for the early diagnosis of $\mathrm{AD}$ rely on handcrafted features, which are inefficient and cannot fully use implicit information in gait data. We need to automatically extract the implicit features in gait data for the early diagnosis of $\mathrm{AD}$, which is the strength of deep learning. Our gait data is composed of skeleton sequences recognized by the Kinect devices. Traditional deep learning methods such as convolutional networks cannot handle such non-Euclidean data. The ST-GCN proposed by (31) shows an excellent performance in extracting the features from skeleton sequences. We apply it as our basic model to the classification of $\mathrm{AD}$ and propose attention-based spatial temporal graph convolutional networks (AST-GCN) according to our task and data characteristics. Based on clinical experience and experimental comparison results, we found that different body parts have different importance in the classification of $\mathrm{AD}$. For this reason, given skeleton sequences, we first perform key point filtering to form our keypoint skeleton sequences and then input it into the proposed attention-based spatial temporal graph convolution networks. The extracted spatial and temporal features are finally used for classification. In the next few subsections, we will first briefly introduce ST-GCN, then we will introduce how we do key point filtering and the proposed attention-based spatial temporal graph convolutional networks.

\subsubsection{Spatial Temporal Graph Convolutional Networks}

Firstly, a spatial temporal graph is constructed from skeleton sequences, as shown in Figure 2A. The edges of the spatial temporal graph consist of two parts. One part is the natural 


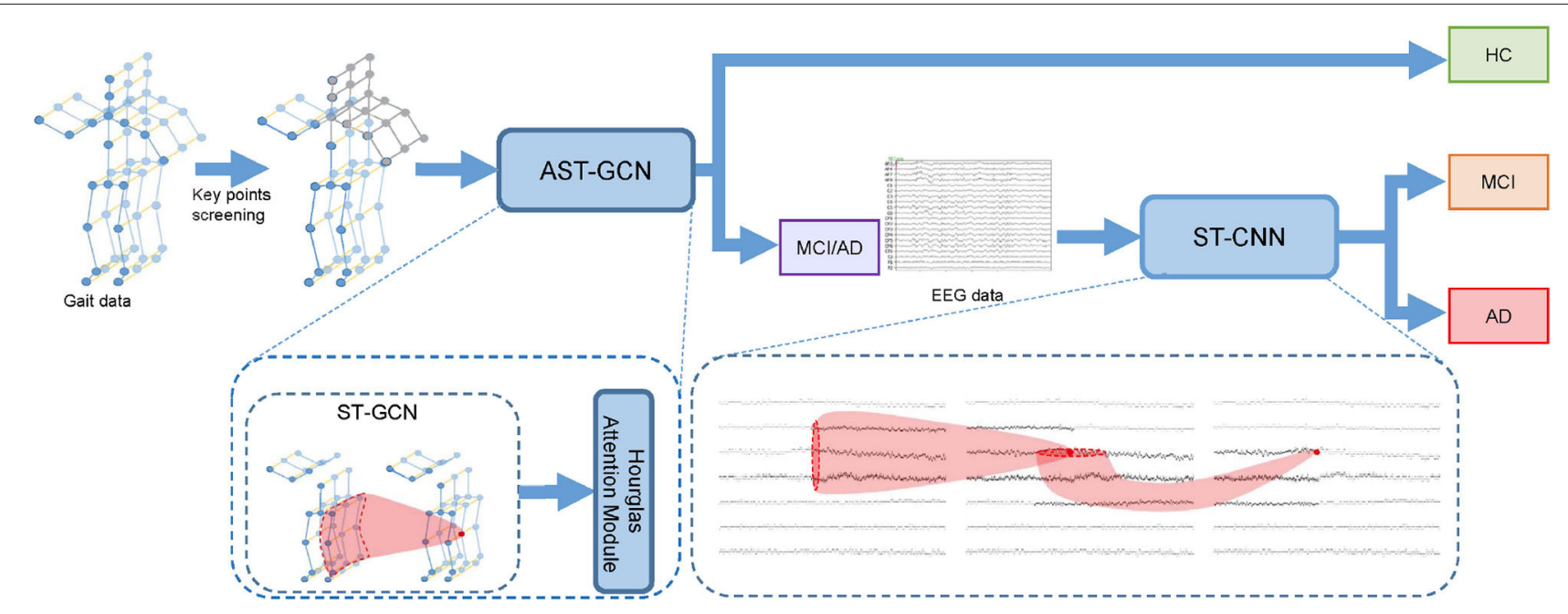

FIGURE 1 | Cascade neural network for the early diagnosis of AD. We perform key point screening on gait data to form key-point skeleton sequences. Then we use attention-based spatial temporal graph convolutional networks (AST-GCN) to extract features and classify the subject into HC or MCl/AD with features. If the subject is classified into $\mathrm{MCl} / \mathrm{AD}$, we will input the EEG data into spatial temporal convolutional networks (ST-CNN) to extract features and perform MCI vs. AD binary classification.

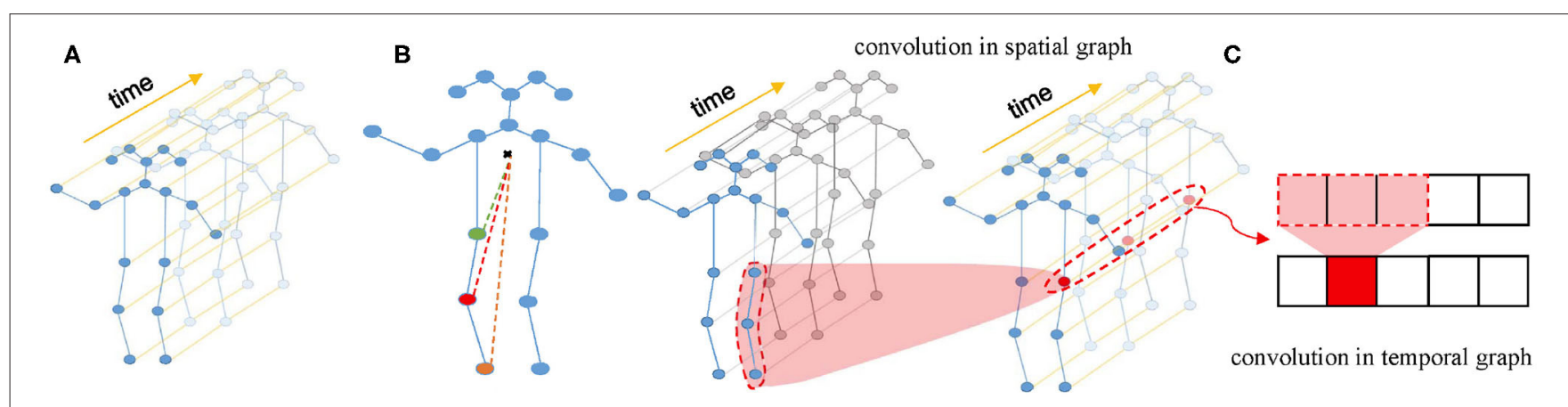

FIGURE 2 | (A) Spatial temporal graph of skeleton sequences. (B) The "Spatial Configuration" strategy. (C) The architecture of ST-GCN.

connections between joint points of the human skeleton in a single frame called spatial edges, and the other part is the time edges formed by connecting the same joint points between adjacent frames. Then, the input features composed of the coordinate vectors of the nodes in the graph are inputted into multiple layers of spatial-temporal graph convolution. Defining the weight function of the graph convolution operation can be realized by a variety of strategies for partitioning each node's neighborhood point set. Experiments show that the "Spatial Configuration" strategy, as shown in Figure 2B, works best. According to this strategy, the neighborhood point set of the root node (red node) is divided into three subsets, namely: (1) The root node itself (red node); (2) The centripetal group (orange node): the nodes closer to the gravity center of the skeleton than the root node; (3) centrifugal group (green node): the nodes that are farther from the gravity center of the skeleton than the root node. The formula of space graph convolution can be written as:

$$
f_{\text {out }}=\Lambda^{-\frac{1}{2}}(\mathbf{A}+\mathbf{I}) \Lambda^{-\frac{1}{2}} f_{\text {in }} \mathbf{W},
$$

where $f_{\text {in }}$ denotes the feature map of the clip composed of the coordinates of input skeleton sequences, which is a $D \times T \times V$ matrix, where $D=3$ corresponds to Three coordinates $(x, y, z)$, $T$ represents the time points i.e., the number of frames of the skeleton sequences, $V$ is the number of nodes that constitute the spatial graph in each frame. $\mathrm{W}$ is the weight function; $\Lambda$ is the degree matrix of the spatial graph; $\mathbf{A}$ is the adjacency matrix of the spatial graph; I is the self-connection matrix. Moreover, $\mathbf{M}$ is proposed as a learnable edge weight, which has the same size as the adjacency matrix. It is used in every layer of spatial temporal graph convolution. Then the Equation (1) can be written as:

$$
f_{\text {out }}=\Lambda^{-\frac{1}{2}}((\mathbf{A}+\mathbf{I}) \bigodot \mathbf{M}) \Lambda^{-\frac{1}{2}} f_{\text {in }} \mathbf{W}
$$

where $\odot$ notes the element-wise multiply. Spatial temporal convolution module consists of a convolution in the spatial graph and a convolution in the temporal graph. The structure of spatial temporal convolution module is shown in Figure 2C. 


\subsubsection{Key Points Filtering}

Several studies $(18,20-24,32)$ found that the AD group has significant differences with the $\mathrm{HC}$ group in gait speed, gait cadence, stride et al. This means that the joints of the lower body, such as the ankles, are more critical for the early diagnosis of $\mathrm{AD}$. Besides, Most subjects are right-handed. It is clinically believed that the left hemisphere of right-handed patients is more sensitive to $\mathrm{AD}$ and more likely to be affected. When we observe the learnable parameter $\mathbf{M}$ of the basic model after it converges, we find that the connections among the joint points of the lower body and the right upper limb are given higher weights, which means that these joint points are more important than other parts. Through experimental comparison, we also verified that performance classification with the skeleton sequences composed of the joint points of the lower body and the right upper limb are better than that with the skeleton sequences composed of other parts. Therefore, we select the joint points of the lower body and the right upper limb to form key-point skeleton sequences.

\subsubsection{Hourglass Attention Module}

From the description above, we can see that different parts are of different importance for the early diagnosis of $\mathrm{AD}$. We argue that even in the key-point skeleton sequences we construct, joints in some parts are more important than other parts, such as ankles and wrists. Therefore, to drive the model further focus on important joints, we introduced an hourglass attention module with a structure similar to the attention module in (33). However, we replaced the pooling layer with a convolutional layer in the time domain with a stride of 4 . The structure of the hourglass attention module is shown in Figure 3.

\subsection{Spatial Temporal Convolutional Networks}

Existing deep learning methods that use EEG data for the classification of $\mathrm{AD}$ convert EEG data into frequency domain information, then calculate PSD features and convert them into images. This way will lose the information in the time domain or even the spatial domain, which is essential to capture coherence and synchronization among different brain regions. The EEGnet proposed by (34) extracts the temporal and spatial features of original EEG data to recognize task-state EEG and shows good performance. However, its feature extraction in the spatial domain of EEG data simply uses a convolution layer to map the data to a single value. We believe that this is not able to fully extract the spatial features of EEG data. We propose the spatial temporal convolutional networks to extract features from original EEG data. Each ST-CNN module consists of a spatial convolution layer with a kernel size of $K_{s} \times 1$ and a temporal convolution layer with a kernel size of $1 \times K_{t}$ similar to (31). In this way, the EEG data is alternately convoluted in the space domain and the time domain through multiple ST-CNN layers to fully extract the implicit features in space and time. The structure of spatial temporal convolutional networks is shown in Table $\mathbf{1 .}$

TABLE 1 | The structure of spatial temporal convolutional networks, where $K_{s}$ and $K_{t}$ are the size of the kernel used in the spatial convolution layer and the temporal convolution layer in a ST-CNN module, respectively.

\begin{tabular}{lclccc}
\hline Layer & $\begin{array}{c}\text { Input } \\
\text { channels }\end{array}$ & Operation & Kernel size & Stride & $\begin{array}{c}\text { Output } \\
\text { channels }\end{array}$ \\
\hline 0 & 3 & Batch normalization & - & - & 3 \\
1 & 3 & ST-CNN & $K_{S}=1, K_{t}=33$ & 1 & 4 \\
2 & 4 & ST-CNN & $K_{s}=15, K_{t}=33$ & 4 & 4 \\
3 & 4 & ST-CNN & $K_{S}=C, K_{t}=33$ & 1 & 16 \\
4 & 16 & ST-CNN & $K_{S}=1, K_{t}=33$ & 4 & 8 \\
6 & 8 & Flatten & - & - & $T / 2$ \\
\hline \multirow{2}{*}{ Classifier } & $T / 2$ & Full connection & - & - & $\mathrm{N}$ \\
& $N$ & SoftMax & - & - & $\mathrm{N}$ \\
\hline
\end{tabular}

$C$ is the number of EEG channels. $T$ is the number of time points. $N$ is the number of classes. In the second layer, we use depthwise separable convolutions. In the 2nd and 4th ST-CNN module, we set stride to 4 as the pooling layer. The residual mechanism is used in each ST-CNN module.

TABLE 2 | The grouping criteria for $\mathrm{HC}, \mathrm{MCl}, \mathrm{AD}$.

\begin{tabular}{lccc}
\hline & HC & MCI & AD \\
\hline MOCA & $>30$ & $18 \sim 30$ & $0 \sim 17$ \\
MMSE & $\geq 24$ & $\geq 24$ & $<24$
\end{tabular}

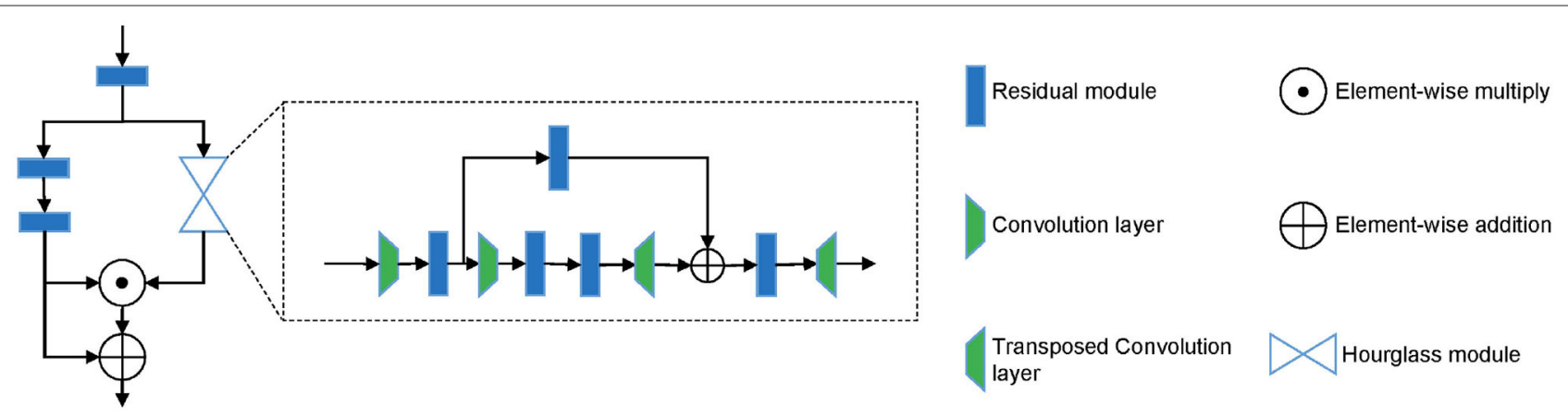

FIGURE 3 | The structure of hourglass attention module. 


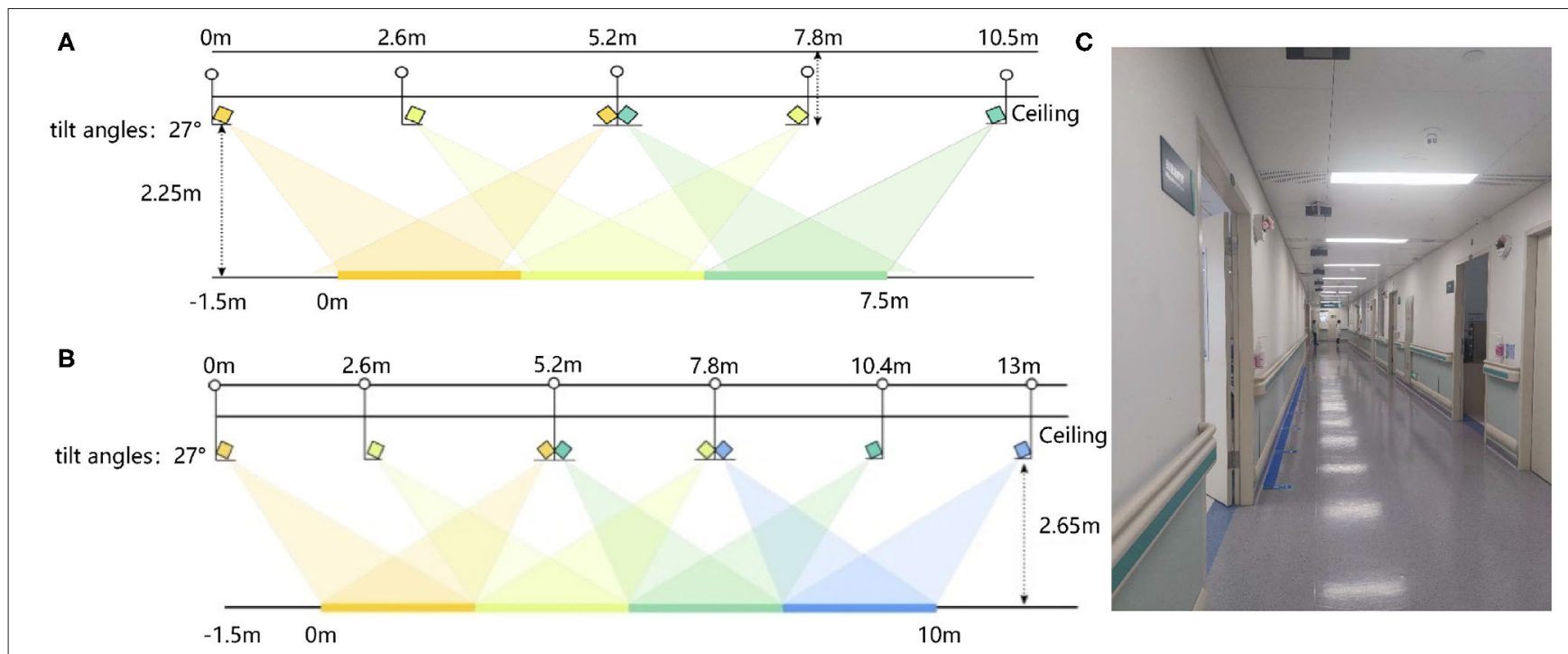

FIGURE 4 | The deployment diagram of Kinect V2.0 devices: (A) The deployment diagram of devices in the Neurology Department. (B) The deployment diagram of devices in the Geriatrics Department. (C) The diagram of the actual data acquisition scene.

\section{EXPERIMENTS}

\subsection{Data Acquisition and Preprocessing}

We collect gait data in cooperation with the Shenzhen Institute of Advanced Technology, Chinese Academy of Sciences and the Shenzhen People's Hospital, and the EEG data are collected by the Shenzhen People's Hospital. All MCI and AD patients are diagnosed by experienced neurologists based on the Montreal Cognitive Assessment(MoCA) and Mini-Mental State Examination (MMSE). We divide the subjects into three groups: $\mathrm{HC}, \mathrm{MCI}$, and $\mathrm{AD}$. These groups include 35 cognitively healthy controls, $35 \mathrm{MCI}$ patients, and $17 \mathrm{AD}$ patients with mildto-severe $\mathrm{AD}$, respectively. The grouping criteria are shown in Table 2. We collect both gait and EEG data for MCI and $\mathrm{AD}$ patients, and only collect gait data for cognitively healthy controls.

\subsubsection{Gait Data}

\subsubsection{Data Acquisition}

Gait data of $52 \mathrm{MCI}$ and $\mathrm{AD}$ patients and 35 control subjects are collected in the Neurology and the Geriatrics Departments of Shenzhen People's Hospital, respectively. Our data collection settings are similar to (35). We use Microsoft Kinect V2.0 cameras as our data acquisition devices. The subjects are asked to walk at their natural and comfortable speed and posture under the devices. They walk a round trip on a straight path about 10 $\mathrm{m}$. We deploy 8 and 6 devices in the Neurology and Geriatrics Department, respectively. The deployment diagram is shown in Figure 4 . The tilt angle of all devices was set $27^{\circ}$.

\subsubsection{Data Preprocessing}

Our gait data consists of the skeleton sequences recognized by the devices. Each skeleton is composed of three-dimensional coordinates of 25 joints. Their indexes are shown in Figure 5A.
In each recording, the devices estimate the skeleton joint coordinates from both the front and back views. However, the skeletons estimated from the back view are less accurate than those from the front view. Therefore, we only select the skeletons from the front view as gait data.

Due to the venue restrictions, the data acquisition devices for patients and the devices for heath controls are deployed in different environments, which may cause differences in absolute coordinates of key points. To eliminate these differences, we follow (36) to perform the following coordinate transformation on the collected gait data in the data preprocessing stage. Since our devices are mounted on the ceiling, and there is an angle of $27^{\circ}$ with the horizontal, we first rotate the coordinates $[x, y, z]$ around the $\mathrm{x}$-axis by $-27^{\circ}$ by calculating

$$
\left|\begin{array}{l}
x^{\prime} \\
y^{\prime} \\
z^{\prime}
\end{array}\right|=\mathbf{R}_{\mathbf{x}} \times\left|\begin{array}{l}
x \\
y \\
z
\end{array}\right| \text {, where } \mathbf{R}_{\mathbf{x}}=\left|\begin{array}{ccc}
1 & 0 & 0 \\
0 & \cos \theta & -\sin \theta \\
0 & \sin \theta & \cos \theta
\end{array}\right|, \theta=-27^{\circ} .
$$

In this way, the skeleton sequences are in a horizontal position relative to the cameras. We then move the origin of the coordinates to the base of the human spine, namely point 0 , by computing

$$
\mathrm{v}_{\tau p}^{\prime}=\mathrm{v}_{\tau p}-\mathrm{v}_{\tau 0}
$$

where $\mathrm{v}_{\tau p}$ is a coordinate vector of $p$ th joint point of the skeleton in $\tau$ th frame. Moreover, the time lengths of gait records are different. Similar to (37), we intercept several clips of data from each gait record through a sliding window to make the number of clip frames consistent. We set the sliding window with a size of 60 frames and a stride of three frames. In this way, we have a total of 5,519 clips, and each gait clip $g_{i}^{j}$ is a matrix with a dimension of $D \times T \times V$, where $D=3, T=60, V=25$. 


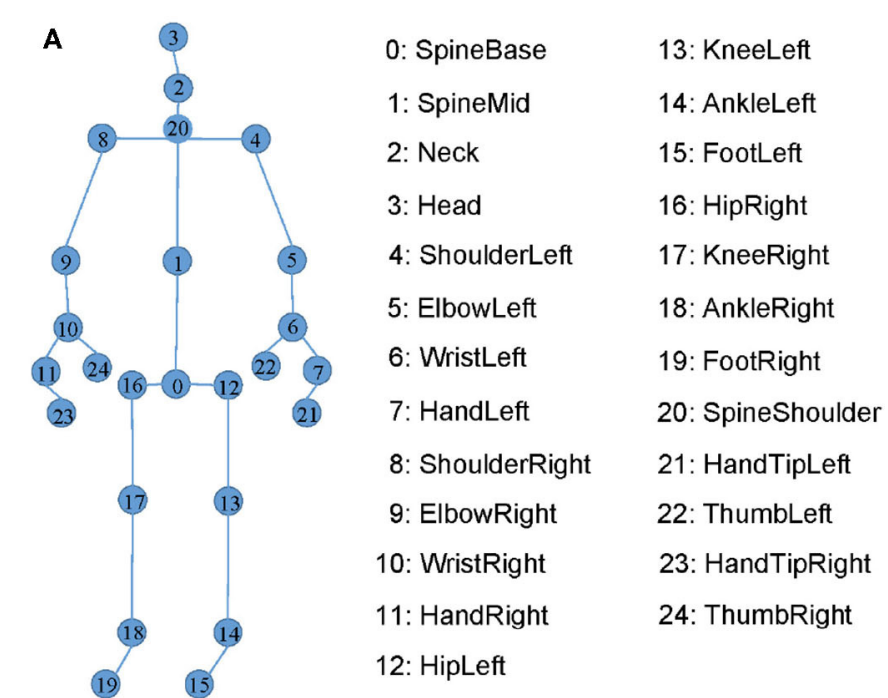

B

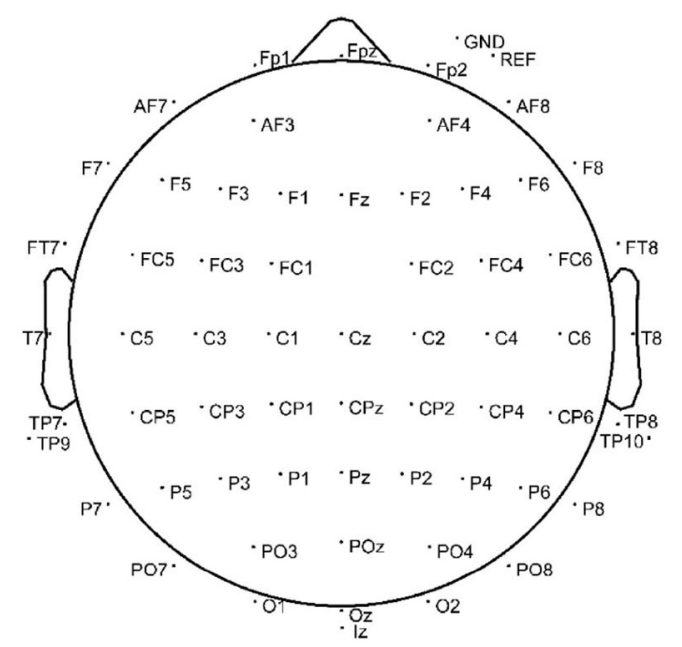

FIGURE 5 | (A) The 25 markers on human skeleton recognized by Kinect. (B) 64 EEG electrode locations in the International 10-20 System.

\subsubsection{EEG Data}

\subsubsection{Data acquisition}

The EEG data are collected by the Neurology Department, Shenzhen People's Hospital. Due to a large mount of artifacts (e.g., myoelectricity) during human walking, the collected EEG data are in low quality. We follow $(6,38)$ to collect higherquality resting EEG data. We collect the EEG data of the patients with eyes closed and with eyes open for 8 min each. We place 64-channel EEG electrodes on the patient's scalp at the standard locations during data acquisition as shown in Figure 5B. The EEG signals are recorded at a sampling frequency of $5,000 \mathrm{~Hz}$.

\subsubsection{Data preprocessing}

After EEG records are collected, we first remove artifacts from EEG records, such as electrooculograms and myoelectricity. Then we re-reference the data. The EEG signals of the Ref and Gnd electrodes are removed, and the average value of the remaining 62 channels is used as a reference value to recalculate the value of the EEG data. Using the original EEG data with a sampling rate of $5,000 \mathrm{~Hz}$ in our ST-CNN will inevitably incur large computation cost. Specifically, the input size is $5,000 \times 62$ when the epoch duration is set to one second. In this paper, we follow Toll et al. (38) to downsample the EEG data to $250 \mathrm{~Hz}$, aiming to reduce the computation cost and improve the inference speed. Similar to (7), we then intercept 120 epochs from each subject's EEG data by a sliding window without overlapping. We set the sliding window with a size of 256 , which is about $1 \mathrm{~s}$. The epochs sampled from the data collected with the eyes open and the eyes closed are concatenated in the time dimension. Finally, we copy it for three times in depth dimension. In this way, we have a total of 5,519 epochs, and each epoch $\varepsilon_{i}^{e}$ is a $3 \times C \times 2 T$ matrix, where $C=62$ is the number of channels of EEG data, $T=256$ denotes the number of time points.

\subsection{Implementation Details}

We randomly select $75 \%$ of the subjects. We use their corresponding data clips as our training set, including 3,277 data clips. The remaining data clips serve as our test set, including 2,242 data clips. We train the model for 50 epochs, using a stochastic gradient descent (SGD) optimizer with an initial learning rate of 0.05 and a batch size of 64 . All experiments are conducted on a single GTX 1060 GPU.

As for EEG data, we randomly select $75 \%$ of the EEG epochs as our training set, containing 4,680 epochs, and the remaining EEG epochs serve as our test set, including 1,560 epochs. We train the model for 70 epochs, using a stochastic gradient descent optimizer with an initial learning rate of 0.005 , and with a batch size of 64. All experiments are conducted on a single GTX 1060 GPU.

\subsection{Comparisons With Other AD Diagnosis Methods}

We compare our proposed method with other existing methods. The results is listed in Table 3. Firstly, we compare our proposed attention-based spatial temporal graph convolutional networks with the methods using handcrafted features. We extract the same features as (10) from gait data and feed them into a SVM classifier with the Gaussian (RBF) kernel and a random forest classifier, respectively. The accuracy of the two classifiers are much lower than our proposed attention-based spatial temporal graph convolutional networks (93.09\%). These results demonstrate that our proposed attention-based spatial Temporal graph convolutional networks is able to extract more powerful features for the diagnosis of $\mathrm{AD}$.

Then we compare the proposed spatial temporal convolutional networks with several baselines on the collected EEG dataset. The baselines include EEGnet (34), ResNet-18 (39), VGG-13 (40), and the standard convolution networks. standard 
TABLE 3 | Comparison with other methods.

\begin{tabular}{|c|c|c|c|c|c|}
\hline \multirow{2}{*}{ Methods } & \multicolumn{2}{|c|}{ Data } & \multicolumn{3}{|c|}{ Accuracy } \\
\hline & Gait & EEG & HC vs. MCI/AD (\%) & $\mathrm{MCl}$ vs. AD (\%) & Three-way classification (\%) \\
\hline Handcrafted features + SVM & $\checkmark$ & & 63.64 & 57.73 & 55.45 \\
\hline Handcrafted feature + RF & $\checkmark$ & & 81.82 & 57.14 & 68.18 \\
\hline AST-GCN(ours) & $\checkmark$ & & 93.09 & 58.41 & 68.51 \\
\hline standard CNN & & $\checkmark$ & - & 69.66 & - \\
\hline EEGnet & & $\checkmark$ & - & 97.85 & - \\
\hline ResNet 18 & & $\checkmark$ & - & 97.59 & - \\
\hline VGG 13 & & $\checkmark$ & - & 96.48 & - \\
\hline ST-CNN(ours) & & $\checkmark$ & - & 98.63 & - \\
\hline cascade neural network(ours) & $\checkmark$ & $\checkmark$ & 93.09 & 98.63 & 91.07 \\
\hline
\end{tabular}

Standard CNN represents the model we substitute 2D convolution layers with a kernel size of $K_{S} \times K_{t}$ for ST-CNN modules. "Handcrafted features + SVM" and "Handcrafted features + $R F "$ indicate the methods using different classifiers with the handcrafted features same as (10). The bold values indicates the best performance that method obtain in that experiment.

TABLE 4 | Ablation study of key point filtering and hourglass attention module on gait data.

\begin{tabular}{lccc}
\hline \multicolumn{2}{c}{ Components } & \\
\cline { 1 - 2 } Key point filtering & Hourglass attention module & Accuracy (\%) \\
\hline$\times$ & $\times$ & 88.18 \\
$\checkmark$ & $\times$ & 91.97 \\
$\times$ & $\checkmark$ & 90.14 \\
$\checkmark$ & $\checkmark$ & 93.09 \\
\hline
\end{tabular}

convolutional networks share the same architecture as the spatial temporal convolutional networks but all ST-CNN modules are replaced with $2 \mathrm{D}$ convolution layers with a kernel size of $K_{s} \times K_{t}$. It is observed that our model achieves the best performance on our data set. We believe that the reason is that ST-CNN can extract the spatial and temporal features from EEG data better. Finally, we test our proposed neural network on our test set. The accuracy of binary classification is $93.09 \%$, and the accuracy of the three-way classification is $91.07 \%$. In addition, we introduce a voting mechanism to improve the fault tolerance of the entire framework. We randomly select a subject $s_{i}$ from the test set and input his gait clip set $\mathcal{G}_{i}$ into AST-GCN for classification. If more than $50 \%$ of the clips are classified into $\mathrm{MCI}$ and $\mathrm{AD}$, all the EEG epochs in $\mathcal{E}_{i}$ will be inputted into ST-CNN to perform binary classification of MCI vs. AD. Otherwise, $s_{i}$ is finally classified into HC. If more than half of the epochs are classified into $\mathrm{MCI}(\mathrm{AD})$, then $s_{i}$ is finally classified into $\mathrm{MCI}(\mathrm{AD})$. With the voting mechanism, the framework can achieve an accuracy of $100 \%$ on the binary classification of $\mathrm{HC}$ vs. MCI/AD, and accuracy of $99.14 \%$ on the three-way classification of $\mathrm{HC}, \mathrm{MCI}$, and $\mathrm{AD}$.

\subsection{Ablation Studies}

\subsubsection{The Effectiveness of the Proposed Component} We conduct experiments on gait data to study the effectiveness of key point filtering and the hourglass attention module. In Table 4, we observe that these two components increase the accuracy from 88.18 to $91.97 \%$ and $90.14 \%$, respectively. With both components, we achieve the best performance with an accuracy rate of $93.09 \%$. We believe the reason is that both components can guide the model to focus more on the points more critical to the diagnostic task. Key point filtering removes insignificant points and noise points, and the attention module drives the model to further focus on the important points in key points.

\subsubsection{Which Key Points Are Essential for AD Diagnosis?}

In Figure 6A, we compare the performance of the skeleton sequences of the lower body, the upper body, and the whole body. We find that the whole body joint performs best. We consider that this is because all joints can provide more information for diagnosis. In addition, we observe that the lower body joints perform better than upper body joints. We believe the reason is that the behavior of lower body is more relative to early $\mathrm{AD}$ diagnosis.

Clinically, it is believed that the left hemisphere of righthanded patients is more sensitive and easier to be affected by $\mathrm{AD}$. As the left hemisphere controls the movement of the right body part, for the right-handed patients, their behaviors of the right body part may provide more information for $\mathrm{AD}$ diagnosis. To study this empirically, we further divide the body joints into two more fine-grained groups, namely "lower body + right upper limb" and "lower body + left upper limb." All subjects in the collected dataset are right-handed. In Figure 6B, "lower body + right upper limb" performs best. these results are consistent with the clinical perspective. Based on such observation, we select the skeleton sequence of "lower body + right upper limb" as a default setting in all experiments.

\subsubsection{Where Should We Use the Hourglass Attention Module?}

We explore the performance of our model with different placements of the attention module. We try to add the hourglass attention module after the third, sixth, and ninth layer of the basic model, respectively, and add three hourglass attention modules after the 3rd, 6th, and 9th layers. The experimental results are shown in Table 5. We see that using three attention modules additionally includes $67.78 \%$ parameters more than 
A

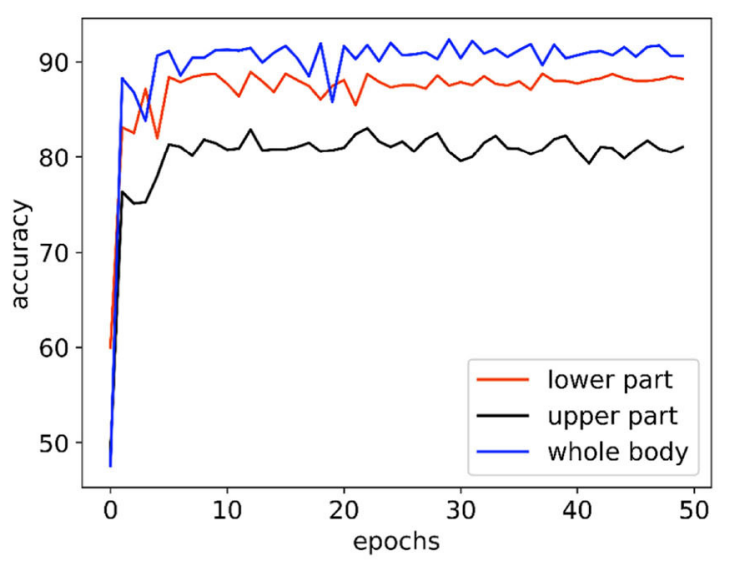

B

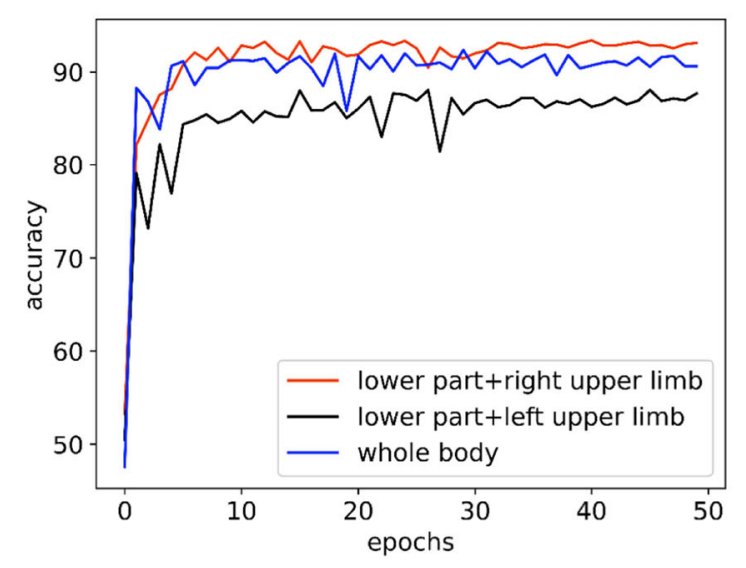

FIGURE 6 | The performance comparison of the basic model on the skeleton sequences composed of different parts: (A) The performance of the basic model on the skeleton sequences composed of the lower body, the upper body, and the whole body. (B) The performance of the basic model on the datasets of skeleton sequences composed of the whole body, the lower body + the right upper limb, and the lower body + the left upper limb.

TABLE 5 | Performance comparison of the models with different hourglass attention module locations.

\begin{tabular}{lccccc}
\hline & Basic model (\%) & After 3rd layer (\%) & After 6th layer (\%) & After 9th layer & After 3rd,6th,9th layers (\%) \\
\hline Accuracy & 88.18 & 88.76 & 88.22 & 90.14 & 87.97 \\
\hline
\end{tabular}

The bold values indicates the best performance that method obtain in that experiment.

TABLE 6 | Comparison of the performance and inference speed with different models.

\begin{tabular}{|c|c|c|c|c|}
\hline \multicolumn{2}{|c|}{ Cascade stage } & \multirow{2}{*}{ Accuracy(\%) } & \multirow{2}{*}{ No. of parameters } & \multirow{2}{*}{ Inference speed (ms) } \\
\hline Stage 1 & Stage 2 & & & \\
\hline AST-GCN (gait) & AST-GCN (gait) & 74.46 & $9.42 \mathrm{M}$ & 7.06 \\
\hline AST-GCN (gait) & ST-CNN (EEG) & 91.07 & 4.72M(4.71M+0.01M) & 3.99 \\
\hline
\end{tabular}

The bold values indicates the best performance that method obtain in that experiment.

using one attention module while decreasing the performance. It is worth nothing that the model with three attention modules outperforms that with one attention module (99.75 vs. $98.04 \%$ ) in the training phase, but it leads to a worse accuracy ( 87.97 vs. $90.14 \%)$ in the testing phase. We conjecture that adding three attention modules may incur the overfitting issue since a larger network is more likely to lead to overfitting in the case of a limited amount of data (41). We see that adding one attention module after the ninth layer of the basic model achieves the best performance. Therefore, we use the model with an attention module after 9 th as the default setting.

\subsubsection{The Efficiency of Our Method}

We conduct an ablation study to validate the effectiveness and efficiency of our method. We replace ST-CNN (classification model with EEG data) in our cascade network with AST-GCN (classification model with gait data). The experimental results are shown in Table 6. Our proposed method with two models significantly outperforms the baseline with one modal (i.e., gait data) while enjoying a faster inference speed (3.99 vs. $7.06 \mathrm{~ms}$ ) and less parameters $(4.72$ vs. $9.42 \mathrm{M})$. Since we do not have the EEG data collected from $\mathrm{HC}$ regarding the difficulty of collecting them in our experimental environments, we did not compare our method with the EEG-based method, and we leave it for our future work.

\section{CONCLUSION}

In this paper, we have exploited both the gait and EEG data to achieve a faster and more accurate classification of AD. To this end, we have proposed a cascade neural network. Our proposed neural network consists of two parts. In the first part, we used gait data to distinguish $\mathrm{HC}$ from patients. For the purpose of modeling the natural connection among the human joints, we have proposed attention-based spatial temporal graph convolutional networks to extract features to classify the HC and patients. In the second part, we further classify MCI and AD patients with EEG data. Compared with the methods that convert EEG data into the frequency domain, we extract the spatial and temporal features from the original EEG data to distinguish the AD patients from MCI patients. The proposed cascade network has the following advantages: (1) The EEG data from HC are not required in our method, which saves a lot of data collection time. (2) The accuracy of our proposed framework in the three-way 
classification of $\mathrm{HC}, \mathrm{MCI}$, and $\mathrm{AD}$ is $91.07 \%$, which is much higher than the method using one modal only (68.18\%), and the accuracy in the binary classification of $\mathrm{HC}$ vs. $\mathrm{MCI} / \mathrm{AD}$ reaches $93.09 \%$. It would be interesting to extend this framework to the diagnosis task of other neurological diseases, and we leave it for future work.

\section{DATA AVAILABILITY STATEMENT}

All datasets generated for this study are included in the article/supplementary material.

\section{ETHICS STATEMENT}

The studies involving human participants were reviewed and approved by Shenzhen People's Hospital Medical Ethics

\section{REFERENCES}

1. Brookmeyer R, Johnson E, Ziegler-Graham K, Arrighi HM. Forecasting the global burden of Alzheimer's disease. Alzheimer's Dement. (2007) 3:186-91. doi: 10.1016/j.jalz.2007.04.381

2. Patterson CA. World Alzheimer Report 2018. London: Alzheimer's Disease International (2018).

3. Wang WH, Hsu YL, Pai MC, Wang CH, Wang CY, Lin CW, et al. Alzheimer's disease classification based on gait information. In: 2014 International Joint Conference on Neural Networks (IJCNN). Beijing (2014) p. 3251-7. doi: 10.1109/IJCNN.2014.6889762

4. Varatharajan R, Manogaran G, Kumar PM, Sundarasekar R. Wearable sensor devices for early detection of Alzheimer disease using dynamic time warping algorithm. Cluster Comput. (2017) 21:681-90. doi: 10.1007/s10586-017-0977-2

5. Gao H, Liu C, Li Y, Yang X. V2VR: reliable hybrid-network-oriented V2V data transmission and routing considering RSUs and connectivity probability. IEEE Trans Intell Transport Syst. (2020). doi: 10.1109/TITS.2020.2983835. [Epub ahead of print].

6. Ieracitano C, Mammone N, Bramanti A, Hussain A, Morabito FC. A Convolutional Neural Network approach for classification of dementia stages based on 2D-spectral representation of EEG recordings. Neurocomputing. (2019) 323:96-107. doi: 10.1016/j.neucom.2018.09.071

7. Bi X, Wang H. Early Alzheimer's disease diagnosis based on EEG spectral images using deep learning. Neural Netw. (2019) 114:119-35. doi: 10.1016/j.neunet.2019.02.005

8. Bennasar M, Setchi R, Hicks Y, Bayer A. Cascade classification for diagnosing dementia. In: 2014 IEEE International Conference on Systems, Man, and Cybernetics (SMC). San Diego, CA (2014) p. 2535-40. doi: 10.1109/SMC.2014.6974308

9. Ning Z, Zhang $\mathrm{K}$, Wang $\mathrm{X}$, Guo $\mathrm{L}$, Hu $\mathrm{X}$, Huang $\mathrm{J}$, et al. Intelligent edge computing in internet of vehicles: a joint computation offloading and caching solution. IEEE Trans Intell Transport Syst. (2020). doi: 10.1109/TITS.2020.2997832. [Epub ahead of print].

10. Seifallahi M, Soltanizadeh H, Mehraban AH, Khamseh F. Alzheimer's disease detection using skeleton data recorded with Kinect camera. Cluster Comput. (2019) 23:1469-81. doi: 10.1007/s10586-019-03014-z

11. Yu Y, Liu S, Guo L, Yeoh PL, Vucetic B, Li Y. CrowdR-FBC: a distributed fogblockchains for mobile crowdsourcing reputation management. IEEE Intern Things J. (2020) 7:8722-35. doi: 10.1109/JIOT.2020.2996229

12. Anderer P, Saletu B, Klöppel B, Semlitsch HV, Werner H. Discrimination between demented patients and normals based on topographic EEG slow wave activity: comparison between $\mathrm{z}$ statistics, discriminant analysis and artificial neural network classifiers. Electroencephalogr Clin Neurophysiol. (1994) 91:108-17. doi: 10.1016/0013-4694(94)90032-9
Committee. The patients/participants provided their written informed consent to participate in this study.

\section{AUTHOR CONTRIBUTIONS}

All authors listed have made a substantial, direct and intellectual contribution to the work, and approved it for publication.

\section{FUNDING}

This research was funded by the Science and Technology Planning Project of Shenzhen Municipality (JCYJ20170818111012390), Sanming Project of Medicine in Shenzhen (SYJY201905 and SYJY201906), and the Shenzhen Health Committee Project (SZXJ2017034).

13. Pritchard WS, Duke DW, Coburn KL, Moore NC, Tucker KA, Jann MW, et al. EEG-based, neural-net predictive classification of Alzheimer's disease versus control subjects is augmented by non-linear EEG measures. Electroencephalogr Clin Neurophysiol. (1994) 91:118-30. doi: 10.1016/0013-4694(94)90033-7

14. Ning Z, Dong P, Wang X, Hu X, Guo L, Hu B, et al. Mobile edge computing enabled $5 \mathrm{G}$ health monitoring for internet of medical things: a decentralized game theoretic approach. IEEE J Select Areas Commun. (2020). [Epub ahead of print].

15. Trambaiolli LR, Lorena AC, Fraga FJ, Kanda PAM, Anghinah R, Nitrini R. Improving Alzheimer's disease diagnosis with machine learning techniques. Clin EEG Neurosci. (2011) 42:160-5. doi: 10.1177/155005941104200304

16. Rossini PM, Buscema M, Capriotti M, Grossi E, Babiloni C. Is it possible to automatically distinguish resting EEG data of normal elderly vs. mild cognitive impairment subjects with high degree of accuracy? Clin Neurophysiol. (2008) 119:1534-45. doi: 10.1016/j.clinph.2008.03.026

17. Gao H, Xu Y, Yin Y, Zhang W, Li R, Wang X. Context-aware QoS prediction with neural collaborative filtering for internet-of-things services. IEEE Intern Things J. (2020) 7:4532-42. doi: 10.1109/JIOT.2019.2956827

18. Callisaya ML, Launay CP, Srikanth V, Verghese J, Allali G, Beauchet O. Cognitive status, fast walking speed and walking speed reserve-the Gait and Alzheimer Interactions Tracking (GAIT) study. GeroScience. (2017) 39:231-9. doi: 10.1007/s11357-017-9973-y

19. Ning Z, Zhang K, Wang X, Obaidat MS, Guo L, Hu X, et al. Joint computing and caching in 5G-envisioned internet of vehicles: a deep reinforcement learning-based traffic control system. IEEE Trans Intell Transport Syst. (2020). doi: 10.1109/TITS.2020.2970276. [Epub ahead of print].

20. Beauchet O, Launay CP, Sekhon H, Montembeault M, Allali G. Association of hippocampal volume with gait variability in pre-dementia and dementia stages of Alzheimer disease: results from a cross-sectional study. Exp Gerontol. (2019) 115:55-61. doi: 10.1016/j.exger.2018.11.010

21. Elbaz A, Artaud F, Singh-Manoux A, Dumurgier J. Gait speed and decline in gait speed as predictors of incident dementia. Innov Aging. (2017) 1:75. doi: 10.1093/geroni/igx004.310

22. Ardle RM, Morris R, Wilson JB, Galna B, Thomas AJ, Rochester LR. What can quantitative gait analysis tell us about dementia and its subtypes? A structured review. J Alzheimer's Dis. (2017) 60:1295-312. doi: 10.3233/JAD-170541

23. Morris R, Lord S, Lawson RA, Coleman S, Galna B, Duncan GW, et al. Gait rather than cognition predicts decline in specific cognitive domains in early Parkinson's disease. J Gerontol Ser A. (2017) 72:1656-62. doi: 10.1093/gerona/glx071

24. Hsu YL, Chung PC, Wang WH, Pai MC, Wang CY, Lin CW, et al. Gait and balance analysis for patients with Alzheimer's disease using an inertialsensor-based wearable instrument. IEEE J Biomed Health Informatics. (2014) 18:1822-30. doi: 10.1109/JBHI.2014.2325413 
25. Gao H, Kuang L, Yin Y, Guo B, Dou K. Mining consuming behaviors with temporal evolution for personalized recommendation in mobile marketing apps. Mobile Netw Appl. (2020) 25:1233-48. doi: 10.1007/s11036-020-01535-1

26. Wang X, Ning Z, Guo S. Multi-agent imitation learning for pervasive edge computing: a decentralized computation offloading algorithm. IEEE Trans Parallel Distrib Syst. (2020) 32:411-25. doi: 10.1109/TPDS.2020.3023936

27. Yu Y, Liu S, Yeoh P, Vucetic B, Li Y. LayerChain: a hierarchical edge-cloud blockchain for large-scale low-delay IIoT applications. IEEE Trans Indus Informatics. (2020) doi: 10.1109/TII.2020.3016025. [Epub ahead of print].

28. Yan JH, Rountree SD, Massman PJ, Doody R, Li H. Alzheimer's disease and mild cognitive impairment deteriorate fine movement control. J Psychiatr Res. (2008) 42:1203-12. doi: 10.1016/j.jpsychires.2008.01.006

29. Choi JS, Oh HS, Kang DW, Mun KR, Choi MH, Lee SJ, et al. Comparison of gait and cognitive function among the elderly with Alzheimer's disease, mild cognitive impairment and healthy. Int J Precis Eng Manufact. (2011) 12:169-73. doi: 10.1007/s12541-011-0024-9

30. Bashivan P, Rish I, Yeasin M, Codella N. Learning representations from EEG with deep recurrent-convolutional neural networks. CoRR. (2016) abs/1511.06448.

31. Yan S, Xiong Y, Lin D. Spatial temporal graph convolutional networks for skeleton-based action recognition. In: AAAI. New Orleans, LA (2018).

32. Wang $X$, Ning Z, Guo S, Wang L. Imitation learning enabled task scheduling for online vehicular edge computing. IEEE Trans Mobile Comput. (2020). doi: 10.1109/TMC.2020.3012509. [Epub ahead of print].

33. Yang Z, Li Y, Yang J, Luo J. Action recognition with spatio-temporal visual attention on skeleton image sequences. IEEE Trans Circuits Syst Video Technol. (2019) 29:2405-15. doi: 10.1109/TCSVT.2018.2864148

34. Lawhern V, Solon AJ, Waytowich NR, Gordon SM, Hung CP, Lance B. EEGNet: a compact convolutional network for EEG-based brain-computer interfaces. J Neural Eng. (2018) 15:056013. doi: 10.1088/1741-2552/aace8c

35. Chattopadhyay P, Sural S, Mukherjee J. Frontal gait recognition from occluded scenes. Pattern Recogn Lett. (2015) 63:9-15. doi: 10.1016/j.patrec.2015.06.004
36. Fang J, Wang T, Li C, Hu X, Ngai ECH, Seet BC, et al. Depression prevalence in postgraduate students and its association with gait abnormality. IEEE Access. (2019) 7:174425-37. doi: 10.1109/ACCESS.2019.295 7179

37. Beyrami SMG, Ghaderyan P. A robust, cost-effective and non-invasive computer-aided method for diagnosis three types of neurodegenerative diseases with gait signal analysis. Measurement. (2020) 156:107579. doi: 10.1016/j.measurement.2020.107579

38. Toll RT, Wu W, Naparstek S, Zhang Y, Narayan M, Patenaude B, et al. An electroencephalography connectomic profile of posttraumatic stress disorder. Am J Psychiatry. (2020) 177:233-43. doi: 10.1176/appi.ajp.2019.180 80911

39. He K, Zhang X, Ren S, Sun J. Deep residual learning for image recognition. In: 2016 IEEE Conference on Computer Vision and Pattern Recognition (CVPR). Las Vegas, NV (2016) p. 770-8. doi: 10.1109/CVPR.2016.90

40. Simonyan K, Zisserman A. Very deep convolutional networks for large-scale image recognition. CoRR. (2015) abs/1409.1556.

41. Srivastava N, Hinton GE, Krizhevsky A, Sutskever I, Salakhutdinov R. Dropout: a simple way to prevent neural networks from overfitting. J Mach Learn Res. (2014) 15:1929-58.

Conflict of Interest: The authors declare that the research was conducted in the absence of any commercial or financial relationships that could be construed as a potential conflict of interest.

Copyright (C) 2020 You, Zeng, Lan, Ren, You, Shi, Zhao, Guo, Jiang and Hu. This is an open-access article distributed under the terms of the Creative Commons Attribution License (CC BY). The use, distribution or reproduction in other forums is permitted, provided the original author(s) and the copyright owner(s) are credited and that the original publication in this journal is cited, in accordance with accepted academic practice. No use, distribution or reproduction is permitted which does not comply with these terms. 